REVIEW ARTICLE

\title{
Micronutrients in Congestive Heart Failure: An Integrative Review
}

\author{
Jaclyn K Gaulden ${ }^{1 *}$, Susan J Appel² and Victoria Kilcawley ${ }^{3}$ \\ ${ }^{1}$ Anderson University, South Carolina, USA \\ ${ }^{2}$ Capstone College of Nursing, The University of Alabama, USA \\ ${ }^{3}$ Spartanburg Regional Healthcare System, South Carolina, USA
}

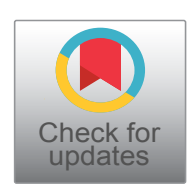

*Corresponding author: Jaclyn K Gaulden, DNP, NP-C, Anderson University, 23 Flintwood Drive, Simpsonville, Anderson, SC 29681, USA, E-mail: jgaulden@gmail.com

Congestive heart failure (CHF) is a common disabling disease that affects approximately 5.1 million people in the United States (US). More than 55,000 deaths annually are contributed to $\mathrm{CHF}[1,2]$. CHF occurs equally among men and women and is responsible for approximately 11 million office visits per year [2]. Economic cost of CHF in 2010 was $\$ 39.2$ billion and accounted for $5.4 \%$ of the total health care budget $[3,4]$.

Hallmark pathology of CHF is cardiac dysfunction which leads to congested organs with hypoperfusion of tissues and may occur with or without preserved left ventricular (LV) ejection fraction [5,6]. Common symptoms of CHF include dyspnea, fatigue, and exertional intolerance as well as edema, an audible $S_{3}$ gallop and rales in the lungs [7]. In short, CHF leads to disability, impaired health-related quality of life (HRQL), decreased exercise tolerance and ultimately death.

As a systemic illness CHF is affected by many factors including neurohormonal abnormalities, elevated levels of inflammation, oxidative stress, and micronutrient deficiencies. Micronutrients consist of vitamins and minerals needed for energy and normal body function. Several micronutrients also have antioxidant properties, reduce inflammation and may also be involved in neurohormonal signaling [3]. Micronutrient supplementation allows for the correction of deficiencies in critical myocyte pathways, including those associated with adenosine triphosphate production (ATP), protein production, intracellular calcium balance, and the reduction of oxidative stress [4].

Advanced age, frequent hospitalizations, anorexia, and malnutrition have all been linked to the increased risk of nutrition deficiencies in patients with CHF. Nutritional status is critical in patients with CHF due to the side effect of unintentional weight loss known as cachexia and decrease of micronutrients from diuretics [3,8-10]. Cachexia often complicates CHF and leads to low albumin as is often seen among patients with poor nutrition [11]. Social isolation among the elderly caused by reduced mobility can also lead to inadequate intake due to eating alone and difficulty preparing meals $[9,10,12]$.

Treatment of CHF includes pharmacologic therapy such as beta-blockers, angiotensin receptor blockers, diuretics, angiotensin-converting enzymes inhibitors and nonpharmacologic therapy, which includes exercise training, implantable cardioverter-defibrillators, cardiac resynchronization therapy, and patient education. Both pharmacologic and nonpharmacologic treatments have shown to have beneficial outcomes and some improvement in mortality; however, hospitalizations for CHF continue to rise $[4,9,13,14]$. Additional interventions are needed to prevent progression and improve outcomes among patients living with CHF.

\section{Methods}

An integrative literature review was conducted using PRISM guidelines, databases were searched (e.g., PubMed, Cumulative Index to Nursing and Allied Health Literature \{CINHAL\}, Cochran, and Google Scholar) for studies published within 2004-2014, that examined the role of micronutrients in patients with CHF. Combinations of the following search terms were used: $\mathrm{CHF}$,

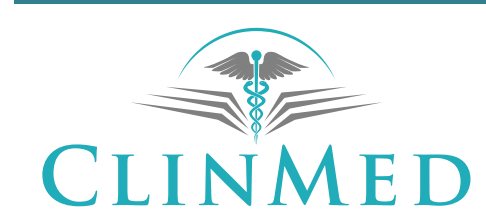

INTERNATIONAL LIBRARY
Citation: Gaulden JK, Appel SJ, Kilcawley V (2018) Micronutrients in Congestive Heart Failure: An Integrative Review. Int Arch Nurs Health Care 4:095. doi.org/10.23937/2469-5823/1510095 Accepted: June 19, 2018: Published: June 21, 2018

Copyright: (c) 2018 Gaulden JK, et al. This is an open-access article distributed under the terms of the Creative Commons Attribution License, which permits unrestricted use, distribution, and reproduction in any medium, provided the original author and source are credited. 


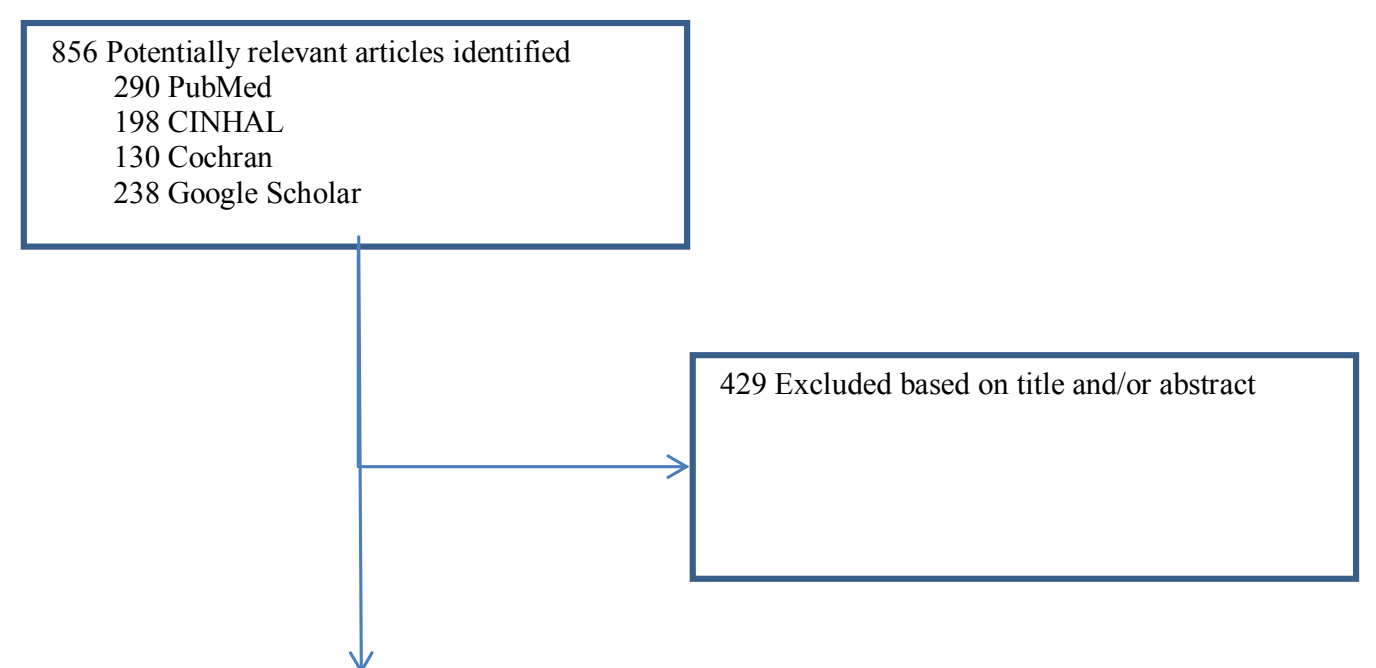

427 Articles identified for title/abstract review

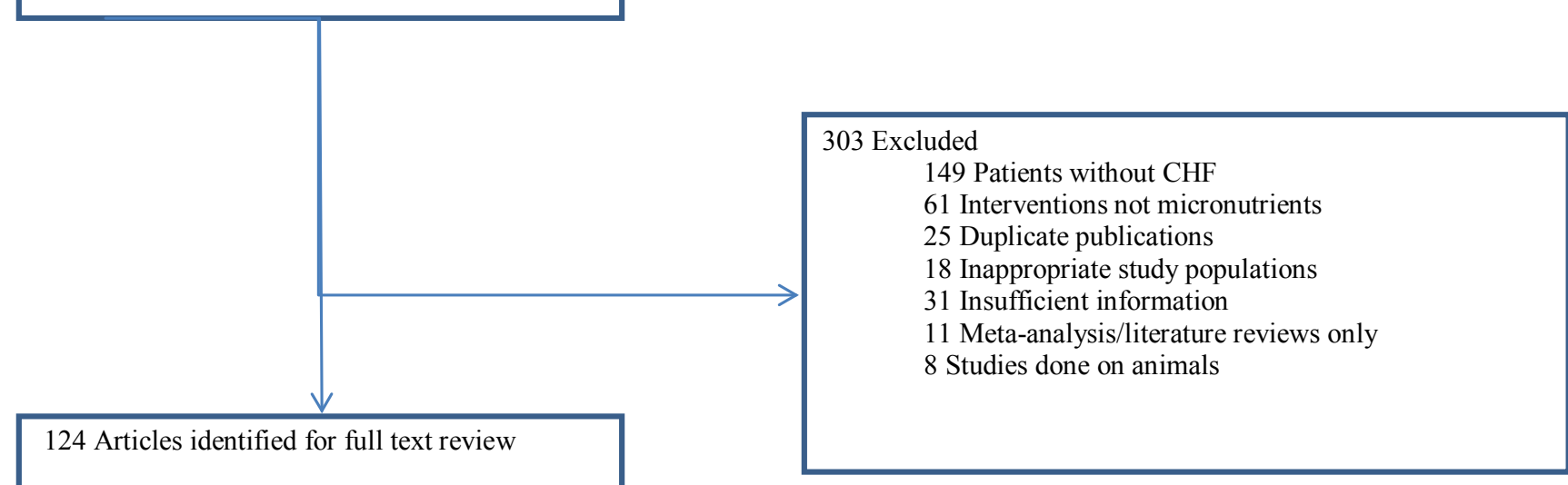

124 Articles identified for full text review

102 Excluded

22 Insufficient information

25 Not a study

48 Not original research

7 Irrelevant information

Figure 1: Flow chart of integrative review.

congestive heart failure, heart failure, micronutrients, nutrition, and $B$ vitamins including thiamine, vitamin $B_{12^{\prime}}$ homocysteine, folate, vitamin $D$, magnesium, and coenzyme $Q_{10}$. Inclusion criteria were articles in English containing original research on participants with CHF aged $\geq 19$. A total of $\mathrm{N}=856$ articles were examined with $\mathrm{n}=$
21 meeting all criterion for inclusion within this review (see Figure 1).

\section{Results}

Studies included in this review are summarized within Table 1. Five studies examined the effects of coenzyme 
$\mathrm{Q}_{10}\left(\mathrm{CoQ}_{10}\right)$ supplementation in patients with $\mathrm{CHF}[7,15-$ 18]. Five studies looked at the role of vitamin $D$ deficiency and supplementation among patients with CHF [19-23]. Six studies examined the role of homocysteinemia as well as treatment with folate, vitamin $\mathrm{B}_{6^{\prime}}$ and vitamin $\mathrm{B}_{12}$ [2429]. In addition, two studies assessed thiamine deficiency and the effect of supplementation $[10,30]$. Two studies examined magnesium oroate supplementation in patients $[14,31]$. One study examined the role of micronutrients on quality of life and LV function [12]. The following sections discuss the different studies for the various micronutrients that play a role in the symptoms and overall cardiac function among patients living with CHF.

\section{$\mathrm{CoQ}_{10}$}

$\mathrm{CoQ}_{10^{\prime}}$ also known as ubiquinone, is a fat-soluble quinone and mitochondrial coenzyme, essential for ATP formation $[15,16,32]$. Studies support that $\mathrm{CoQ}_{10}$ is necessary for adequate LV function [7,15-18]. CoQ $_{10}$ can improve cellular bioenergetics and has potential to prevent and treat CVD, including CHF by increasing LV function [15]. It is estimated that $25 \%$ of the required $\mathrm{CoQ}_{10}$ is obtained from diet. The remaining $75 \%$ is produced within body cells $[32,33]$. Constant supply of $\mathrm{CoQ}_{10}$ is necessary for adequate LV function. However, aging decreases endogenous synthesis of $\mathrm{CoQ}_{10}[17,32]$.

A study by Molyneux, et al. [16] examined the association between $\mathrm{CoQ}_{10}$ levels and increased mortality by following $N=236$ patients with CHF. As 76 deaths occurred leading to a conclusion that low $\mathrm{CoQ}_{10}$ levels increase mortality. The correlation between the $\mathrm{CoQ}_{10}$ and mortality was found to be greater than that with plasma N-terminal peptide of B-type natriuretic peptide (NT-proBNP) suggesting that decreased levels of $\mathrm{CoQ}_{10}$ are associated with increased mortality and LV function regardless of the initial severity of CHF [16].

A 5-year prospective randomized-controlled trial was conducted among elderly by Alehagen, Johansson, Björnstedt, Rosén, and Dahlström [17]. Patients were randomized to receive a combination pill of $\mathrm{CoQ}_{10} 200$ mg per day with selenium yeast 200 mcg per day ( $n=$ $124)$ or placebo $(n=104)$ for 48 months. They were monitored every $6^{\text {th }}$ month for 5 years with clinical examinations of LV function. The researchers concluded a significant reduction in cardiovascular mortality was seen among the treatment group $(p=0.015)$. NT-proBNP levels were lower after supplementation $(p=0.014)$ as well an improved LV function $(p=0.03)$ [17].

Adarsh, Kaur, and Mohan [15] found that $\mathrm{CoQ}_{10}$ had a significant impact on New York Heart Association (NYHA) classifications for CHF, quality of life, diastolic dysfunction, and dysrhythmias. They conducted a quasi-experimental study $(\mathrm{N}=87$ ) with hypertrophic cardiomyopathy. The treatment group received $100 \mathrm{mg}$ of $\mathrm{CoQ}_{10}$ twice daily and the control received conventional treatment. The treatment group had an improvement in: NYHA classification by $\geq 1(p<0.005)$, quality of life on 6 minute walk test $(p<0.005)$, diastolic function by $\geq 1$ parameter $(p<0.005)$, and significant reduction in LVOT gradient $\geq 15 \mathrm{mmHg}$ in obstructive CHF cases ( $p$ $<0.005$ ). Among the treatment group, no patients suffered cardiac dysrhythmias in contrast to four patients in the control having ventricular tachycardia [15].

A randomized-controlled trial conducted by Fumagalli, et al. [7], found that total work capacity (TWC) was significantly improved in patients with stable CHF after treatment with $\mathrm{CoQ}_{10}$ terclatrate (Q-ter) and creatine daily for two months. Patients $(N=67)$ were randomized to receive $320 \mathrm{mg}$ of Q-ter and $340 \mathrm{mg}$ of creatine daily or placebo for eight weeks. The treatment group had improved TWC $(p=0.04)$, with no change in the placebo $(p=0.06)$. Peak $\mathrm{VO}_{2}$ increased in the treatment group ( $p=0.003$ ) with no significant change in placebo. They concluded that when Q-ter and creatine are added to conventional treatment, positive effects on physical performance occur [7].

Belardinelli, et al. [18] studied patients ( $N=23)$ with a NYHA class II and III and stable CHF secondary to ischemic heart disease, by randomizing patients into three groups, who received four different treatments simultaneously that consisted of $\mathrm{CoQ}_{10}, \mathrm{CoQ}_{10}$ plus exercise training, placebo, and placebo plus exercise training in this double-blind, placebo-controlled cross-over design with each phase lasting four weeks. Researchers concluded that $\mathrm{CoQ}_{10}$ was without any adverse effects, could improve LV function, functional capacity, and endothelial function. $\mathrm{CoQ}_{10}$ paired with exercise training resulted in higher levels of $\mathrm{CoQ}_{10}$ and reduced peak systolic wall thickening $(p \leq 0.001)[18]$.

Correction of $\mathrm{CoQ}_{10}$ deficiencies with supplementation has resulted in decreased mortality, improvement in cardiac function, NYHA classification, improvement in quality of life, and total work capacity. No side effects were noted among the studies and all researchers concluded that $\mathrm{CoQ}_{10}$ could safely be given to patients with CHF $[7,15-18]$.

\section{Vitamin D}

Vitamin D deficiency is associated with CVD risk factors. Vitamin $D$ deficiency has been linked to low sun exposure, obesity, hypertension, diabetes, CVD and even death [21,23]. Vitamin D deficiency is common among patients with $\mathrm{CHF}$ due to decreasing plasma renin activity (PRA) levels as it binds to the Vitamin $D$ receptors and preventing renin transcription which is actively involved in CVD [23]. Deficiency of Vitamin D is seen in aging due to a decrease in skin synthesis, with poor exposure to the sun as well as an impairment of 7-dehydrocholesterol conversions to previtamin D. Among patients with CHF, Vitamin D deficiency occurred with those that had a higher NYHA classification, shorter 6-minute walk test, and increased BNP [19]. 
A retrospective review of $\mathrm{CHF}$ patients conducted by Ameri, et al. [19] over months having similar sunny hours. Among the patients, only $2.2 \%(n=2)$ had an adequate laboratory value of vitamin $D(25[\mathrm{OH}] \mathrm{D}$ above $75 \mathrm{nmol} / \mathrm{L}$ ). Due to this high prevalence of vitamin $D$ deficiency, a retrospective review of patients with no diagnosis of $\mathrm{CHF}$ was done $(n=31)$ for comparison. Vitamin D levels were consistently lower in the patients with CHF as opposed to the control [19]. Likewise, a study by Gotsman, et al. [21] concluded that vitamin D deficiency is associated with CHF. In this larger study, ( $N=49,834)$ all members of a Health Maintenance Organization (HMO), aged $\geq 45$ had vitamin $D$ measured. Vitamin D levels among members with CHF were compared to the members without CHF. Patients with CHF had consistently lower levels than that of the control ( $p<0.00001)$. Prevalence of vitamin D deficiency was greater among patients with CHF $(p<0.00001)$. During the follow up period, the mortality rate was $15.4 \%$ ( $n=$ 455/3009) among patients with CHF but, only $1.7 \%$ ( $n=$ $802 / 46,825)(p<0.00001)$ among the control, leading to a conclusion that vitamin $\mathrm{D}$ deficiency is a predictor of overall mortality [21].

Pilz, et al. [22], demonstrated the negative impact of vitamin D deficiency on LV function in the LUCRIC study ( $N=3299)$, in patients referred for cardiac angiography and NT-proBNP, 25(OH) D and 1, 25(OH) D was obtained the morning before coronary angiography. A significant correlation was also seen with impaired LV function and decreased vitamin D levels $(p<0.001$ ) as well as with a higher NYHA classification. During the follow up period of 7.7 years, 760 died with 188 from sudden cardiac death (25\%) and 116 from CHF (15\%). Among the participants, more sudden cardiac and CHF deaths occurred between December and May (101 SCDs and 62 CHF deaths) which were the months that patients had the lowest vitamin D levels at baseline [22].

Multiple studies $[19,21,22]$ have examined vitamin D levels in patients with CHF and concluded that a strong correlation is seen between poor LV function and vitamin $D$ deficiency. It is important to determine if supplementation will improve overall cardiac function. Amin, et al. [20] sought to determine if vitamin D supplementation improves the severity of $\mathrm{CHF}$. This study looked at patients $(N=100)$ with CHF that were on optimal medical treatment for at least three months. Those deficient in vitamin $\mathrm{D}$ were given supplements of vitamin $D_{3}$ (cholecalciferol) 50,000 IU weekly for eight weeks followed by 50,000 IU monthly for two months. After treatment patients were reassessed and the mean concentration of vitamin D increased by $41.86 \mathrm{nmol} / \mathrm{L}$ and Parathyroid Hormone levels decreased by 36.73 ng/L. In addition, a decrease in NT-proBNP levels was noted $(p<0.001)$ and an improved NYHA classification $(p<0.001)$. Vitamin D supplementation improved the 6 -minute walk test $(p<0.001$ ) leading to the conclusion that supplementation decreases the severity of CHF and improves functional capacity [20].

Schroten, et al. [23] conducted a randomized-prospective trial (VitD-CHF trial) with stable patients ( $\mathrm{N}=$ 99) diagnosed with CHF on optimal medical therapy. Patients were randomized to Vitamin $D_{3} 2,000$ IU daily or control without placebo given for six weeks. Results showed in the treatment group an increase in $25(\mathrm{OH})$ D levels and 1,25(OH)2D levels by week 3 with lasting effects throughout the treatment period. At the end of the six week trial period, $52 \%$ of the treatment group had adequate vitamin $D$ levels whereas only $4 \%$ demonstrated normal vitamin D levels in the control. The treatment group had a decrease in PRA levels at the end of the six week period compared to the control $(p=0.002)$ along with PRC $(p=0.020)$ and PTH $(p=0.004)$. They concluded that patients with CHF could safely be treated with supplemental vitamin $\mathrm{D}_{3}$ [23].

\section{B-Vitamins}

A deficiency of B-vitamins is common among patients with $\mathrm{CHF}$ and contributes to the decreased energy reserves. B-vitamins play an important role in cellular energy production; therefore a deficiency of B-vitamins may impair adenosine triphosphate (ATP) synthesis. Due to the diminished energy stores available for myocyte contraction and decreased LV function worsening of CHF is seen [13,34]. A positive correlation between increased plasma homocysteine (homocysteinemia) was linked to the risk of CHF. Homocysteinemia is marker of overall poor prognosis due to increased cytokine production in CHF $[13,27,29]$. Homocysteinemia is negatively correlated with levels of: Creatinine, folate, vita$\min B_{12}$, and serum albumin [27]. A positive correlation was found between homocysteinemia and an increased risk of CHF among healthy people [13].

Rodríguez, et al. [27] evaluated the prevalence, significance, and prognosis of homocysteinemia in hospitalized patients ( $\mathrm{N}=337$ ). In addition, $\mathrm{n}=36$ control $\mathrm{s}$ were used for comparison. Results indicated that $\mathrm{CHF}$ was related to homocysteinemia in contrast to controls without CHF ( $p=0.005)$. Prevalence of CHF correlated with homocysteinemia by $30 \%$ when level was below the $15^{\text {th }}$ percentile, $40 \%$ between the $15^{\text {th }}$ and $50^{\text {th }}$ percentile, and up to $48 \%$ above the $50^{\text {th }}$ percentile [27].

Guéant-Rodriguez, et al. [26] evaluated ( $N=709$ ) for the association between homocysteinemia and LV dysfunction. Results showed that total homocysteinemia was higher in patients with CAD versus those without ( $p$ $<0.001$ ) and higher homocysteinemia was seen in patients with NYHA classification III than II ( $p=0.0009)$. An association was seen between increased homocysteinemia with decreased LV function $(<40 \%)(p<0.0001)$ [26].

Gibelin, et al. [25] examined the impact of homocysteinemia in patients $(\mathrm{N}=278)$ with $\mathrm{CHF}$ versus a controls. It was noted that mean homocysteine levels were high- 
er in the CHF group versus the controls $(p=0.001)$. They concluded there is a correlation between NYHA classification and homocysteine levels as well as homocysteinemia is a predictor of mortality in CHF ( $p=0.0003$ ) [25]. Maurer, et al. [24] concluded that homocysteinemia is an important risk factor for cardiac events among $\mathrm{CHF}$ patients $(N=161)$. It was found that patients with the highest homocysteine levels ( $\geq 20 \mu \mathrm{mol} / \mathrm{L}$ ) had the highest incidence of death $(p<0.035)$ [24].

Lee, et al. [28] sought to determine if supplementation with vitamin- $B_{6}$ had a positive effect on lowering homocysteine levels in patients with CVD by looking at patients $(N=42)$ with at least a $70 \%$ stenosis of a major coronary artery. Patients were randomized to one of five groups: Placebo, vitamin- $B_{6} 5 \mathrm{mg}$, vitamin- $B_{6} 10$ $\mathrm{mg}$, vitamin- $\mathrm{B}_{6} 50 \mathrm{mg}$, or folic acid $5 \mathrm{mg}$ with $0.25 \mathrm{mg}$ vitamin- $B_{12}$ for 12 weeks. Homocysteine levels decreased significantly (32\%) with 12 -weeks of folic acid and vitamin- $B_{12}$ supplementation $(p<0.001)$. Vitamin- $B_{6}$ and placebo were not effective in lowering homocysteine [28].

Andersson, Edvinsson, and Edvinsson [29] conducted a study to determine if vitamin supplementation can normalize homocysteine levels in patients $(N=14)$ with CHF. The homocysteine levels decreased $(p<0.01)$. Therefore, it was concluded that vitamin supplementation with folate can lower homocysteine levels in patients with CHF [29].

Thiamine, a water-soluble B-complex vitamin, plays a multifactorial role in patients with $\mathrm{CHF}[10,35]$. Thiamine acts as a vasodilator and improves LV function by reducing afterload and increasing diuresis. Thiamine deficiency can cause cardiac hypertrophy, dysrhythmias, and worsening CHF $[10,35]$. Humans cannot effectively store or produce thiamine and are therefore dependent on ingestion through supplements or food. Patients with CHF are often deficient in thiamine due to age and comorbid conditions. Dietary issues also contribute to thiamine deficiency with early satiety and cachexia as often seen in patients with $\mathrm{CHF}$ and the need for a low sodium diet [35].

Hanninen, Darling, Sole, Barr, and Keith [10] demonstrated how prevalent thiamine deficiency is for $\mathrm{CHF}$ patients. Among $(\mathrm{N}=100)$ hospitalized CHF patients who were analyzed for a thiamine deficiency and compared with matched controls. Patients admitted with CHF were found to be more likely to have a thiamine deficiency than that of their controls with $33 \%$ having a deficiency $(p=0.007)$ with spironolactone use, lack of thiamine supplementation, and preserved renal function significantly relating to the deficiency. Fifty percent of patients with CHF were also malnourished [10].

In a randomized-controlled, cross over study, Schoenenberger, et al. [30] found that beneficial effects are seen in patients with CHF when given thiamine supplementation. Patients $(\mathrm{n}=9)$ were randomized to receive thiamine $300 \mathrm{mg}$ per day or placebo for 28 days. After 6 weeks, a wash-out period was done to eliminate carry-over effects before a second treatment period of 28 days was started in which the medication was reversed. After twenty-eight days of treatment LV function increased in the thiamine group from $29.5 \%$ to $32.8 \%(p=0.024)$ with no improvement seen in the placebos. Improvement in functional status was seen on the 6-minute walk test when receiving thiamine [30].

\section{Magnesium}

Magnesium (Mg) plays an important role in the heart by its role is electrophysiology of cardiomyocytes. Patients with CHF often have hypomagnesemia and/or intracellular magnesium depletion leading to increased rate of mortality. Hypomagnesiumemia negatively affects heart rate variability which is often reported in CHF and leads to shorter survival [31].

Almozino-Sarafian, et al. [31] examined the effect of $\mathrm{Mg}$ administration on heart rate variability (HRV) in normomagnesemic patients with systolic CHF. Patients (N $=32$ ) were randomly assigned to receive $\mathrm{Mg}$ citrate 300 $\mathrm{mg} /$ day for 5 weeks and compared to controls without placebo. After 5 weeks of treatment, patients receiving the Mg citrate, had an increase in Mg levels $(p<0.001)$ than controls $(p=0.042)$. In contrast, the intracellular $\mathrm{Mg}$ rose in the treatment group ( $\mathrm{p}=0.025$ ) while no statistically significant change occurred in the controls $(p$ $=0.7$ ). . The treatment group had significantly increased HRV. The investigators concluded that supplementation increases serum $\mathrm{Mg}$ levels, intracellular $\mathrm{Mg}$ levels and HRV-CD and can be beneficial in patients with CHF [31].

Stepura \& Martynow [14] evaluated supplemental magnesium orotate on clinical symptoms and mortality in patients with CHF. In this prospective, randomized-controlled study, patients $(\mathrm{N}=79)$ were randomized to receive magnesium orotate $6000 \mathrm{mg}$ for 1 month then $3000 \mathrm{mg}$ for 11 months or placebo. One year survival rates in the treatment group were better compared to controls $(p<0.05)$. Clinical symptoms significantly improved in the treatment group while symptoms deteriorated among the controls $(p<0.001)$ leading to the conclusion that magnesium orotate can be used as additional therapy in patients with $\mathrm{CHF}$ and is beneficial by decreasing symptoms as well as increasing survival rate [14].

\section{Other Micronutrients}

Many other micronutrients have shown promise in the treatment of $\mathrm{CHF}$ including vitamin- $\mathrm{A}$, vitamin- $\mathrm{C}$, vitamin-E, selenium, copper, magnesium, zinc, and L-carnitine $[3,4]$. Witte, et al. [12] conducted a randomized-trial using a two-by-two method to determine the effect of micronutrient supplementation in patients with CHF. Patients $(\mathrm{N}=30)$ were randomized to receive capsules of high dose micronutrients including calcium, magnesium, zinc, copper, selenium, vitamin-A, thiamine, riboflavin, 
vitamin- $B_{6}$, vitamin- $B_{12}$, folate, vitamin- $C$, vitamin- $E$, and $\mathrm{CoQ}_{10}$ or placebo for nine months. The treatment group demonstrated an increase in LV function with no change in controls $(p<0.05)$. The treatment group had a significant improvement in quality of life score in contrast to a slight decrease in the placebo group $(p<0.05)$. The 6-minute walk test, NYHA classification, and cytokine levels remained unchanged in both the treatment and placebo groups. They concluded that micronutrient supplementation could positively impact cardiac function and quality of life in patients with CHF [12].

\section{Discussion}

Many studies have been conducted that concluded that patients with $\mathrm{CHF}$ are often deficient in micronutrients. A deficiency of micronutrients can cause decreased LV function, decreased quality of life, and increased symptoms contributing to a progression of CHF. However, a much smaller number of studies have conducted interventions with micronutrient supplementation. Studies have shown that patients with CHF can safely take micronutrient supplementations and experience a beneficial effect. There is a great need to decrease mortality in patients with CHF since current treatments are not being fully successful.

$\mathrm{CoQ}_{10}$ has been shown to decrease mortality from a cardiac cause, improve NYHA classification, improve quality of life, increased total work capacity, and increase LV ejection fraction [7,15-18]. Likewise, vitamin-D supplementation decreases cardiovascular related mortality, increases LV ejection fraction, improvement of NYHA classification, increase in physical performance, as well as decreased PRA and PRC [19-23]. In addition, homocysteinemia has been shown to negatively impact cardiovascular health. Supplementation with B-vitamins, particularly folate, has been linked to decreasing homocysteine levels and therefore reduce symptoms of $\mathrm{CHF}$ [24-29]. Thiamine can also improve functional status in patients living with $\mathrm{CHF}[10,30]$. Magnesium plays a role in the electrophysiology of the heart and supplementation is beneficial in patients with CHF [14,31].

A limitation of this area of research is a majority of studies were very small with studies ranging from only nine patients to a whole $\mathrm{HMO}(\mathrm{N}=46,825)$. In addition, patients were not followed for an extended period of time with the longest being 7.7 years with a majority of studies being shorter than two years. This leaves room for additional larger and longer studies to be performed. Studies indicate that micronutrients not only help improve LV function but also increase quality of life when living with CHF.

\section{References}

1. Go AS, Mozaffarian D, Roger VL, Benjamin, EJ Berry JD, et al. (2013) Heart disease and stroke statistics-2013 update: A report from the American Heart Association. Circulation 127: e6-e245.
2. Emory Healthcare (2013) Heart failure statistics.

3. McKeag NA, McKinley MC, Woodside JV, Harbinson MT, McKeown PP (2012) The role of micronutrients in heart failure. Journal of the American Academy of Nutrition and Dietetics 112: 870-886.

4. Soukoulis V, Dibu JB, Sole M, Anker SD, Cleland J, et al. (2009) Micronutrient deficiencies an unmet need in heart failure. J Am Coll Cardiol 54: 1660-1673.

5. Borlaug BA, Redfield MM (2011) Diastolic and systolic heart failure are distinct phenotypes within the heart failure spectrum. Circulation 123: 2006-2013.

6. Alsafwah S, LaGuardia SP, Arroyo M, Dockery BK, Bhattacharya SK, et al. (2007) Congestive heart failure is a systemic illness: A role for minerals and micronutrients. Clin Med Res 5: 238-243.

7. Fumagalli S, Fattirolli F, Guarducci L, Cellai T, Baldasseroni $S$, et al. (2011) Coenzyme $Q_{10}$ terclatrate and creatine in chronic heart failure: A randomized, placebo-controlled, double-blind study. Clinical Cardiology 34: 211-217.

8. Keith ME, Walsh NA, Darling PB, Hanninen SA, Thirugnanam S, et al. (2009) B-vitamins deficiency in hospitalized patients with heart failure. J Am Diet Assoc 109: 1406-1410.

9. Krim SR, Campbell P, Lavie CJ, Ventura H (2013) Micronutrients in chronic heart failure. Current Heart Failure $\mathrm{Re}-$ ports 10: 46-53.

10. Hanninen SA, Darling PB, Sole MJ, Barr A, Keith ME (2006) The prevalence of thiamin deficiency is hospitalized patients with congestive heart failure. Journal of the American College of Cardiology 47: 354-361.

11. Araújo JP, Lourenco $P$, Rocha-Goncalves $F$, Ferreira $A$, Bettencourt $P$ (2011) Nutritional markers and prognosis in cardiac cachexia. Int J Cardiol 146: 359-363.

12. Witte KK, Nikitin NP, Parker AC, von Haehling S, Volk H, et al. (2005) The effect of micronutrient supplementation on quality-of-life and left ventricular function in elderly patients with chronic heart failure. European Heart Journal 26: 2238-2244.

13. Azizi-Namini P, Ahmed M, Yan AT, Keith M (2012) The role of $B$ vitamins in the management of heart failure. Nutrition in Clinical Practice 27: 363-374.

14. Stepura OB, Martynow Al (2009) Magnesium orotate in severe congestive heart failure $(\mathrm{MACH})$. Int $\mathrm{J}$ Cardiol 131: 293-295.

15. Adarsh K, Kaur H, Mohan V (2008) Coenzyme $Q_{10}\left(C_{0} Q_{10}\right)$ in isolated diastolic heart failure in hypertrophic cardiomyopathy (HCM). BioFactors 32: 145-149.

16. Molyneux SL, Florkowski CM, George PM, Pillbrow AP, Frampton CM, et al. (2008) Coenzyme $Q_{1}:$ An independent predictor of mortality in chronic heart failure. Journal of the American College of Cardiology 52: 1436-1441.

17. Alehagen $U$, Johansson $P$, Björnstedt $M$, Rosén $A$, Dahlström U (2013) Cardiovascular mortality and N-terminal-proBNP reduced after combined selenium and coenzyme $Q_{10}$ supplementation: A 5-year prospective randomized double-blind placebo-controlled trial among elderly Swedish citizens. International Journal of Cardiology 167: 1860-1866.

18. Belardinelli R, Mucaj A, Lacalaprice F, Solenghi M, Seddaiu $G$, et al. (2006) Coenzyme $Q_{10}$ and exercise training in chronic heart failure. European Heart Journal 27: 26752681. 
19. Ameri P, Ronco D, Casu M, Denegri A, Bovio M, et al. (2010) High prevalence of vitamin D deficiency and its association with left ventricular dilation: An echocardiography study in elderly patients with chronic heart failure. Nutrition, Metabolism, \& Cardiovascular Diseases 20: 633-640.

20. Amin A, Minaee S, Chitsazan M, Naderi N, Taghavi S, et al. (2013) Can vitamin D supplementation improve the severity of congestive heart failure? Congestive Heart Failure 19: E22-E28.

21. Gotsman I, Shauer A, Zwas DR, Hellman Y, Keren A, et al. (2012) Vitamin D deficiency is a predictor of reduced survival in patients with heart failure; vitamin $D$ supplementation improves outcome. Eur J Heart Fail 14: 357-366.

22. Pilz S, Marz W, Wellnitz B, Seelhorst U, Fahrleitner-Pammer A, et al. (2008) Association of vitamin D deficiency with heart failure and sudden cardiac death in a large cross-sectional study of patients referred for coronary angiography. The Journal of Clinical Endocrinology \& Metabolism 93: 3927-3935.

23. Schroten NF, Ruifrok WP, Kleijn L, Dokter MM, Sillje HH, et al. (2013) Short-term vitamin $D_{3}$ supplementation lowers plasma renin activity in patients with stable chronic heart failure: An open-label, blinded end point, randomized prospective trail (VitD-CHF trial). American Heart Journal 166: 357-364.

24. Maurer M, Burri S, de Marchi S, Hullin R, Martinelli M, et al. (2010) Plasma homocysteine and cardiovascular risk in heart failure with and without cardiorenal syndrome. International Journal of Cardiology 141: 32-38.

25. Gibelin P, Serre S, Candito M, Houcher B, Berthier F, et al. (2006) Prognostic value of homocysteinemia in patients with congestive heart failure. Clin Chem Lab Med 44: 813-816.

26. Guéant-Rodriguez RM, Juillière $Y$, Nippert $M$, Abdelmouttaleb I, Herbeth B, et al. (2007) Left ventricular systolic dysfunction is an independent predictor of homocysteine in angiographically documented patients with or without coronary lesions. J Thromb Haemost 5: 1209-1216.

27. Rodríguez JJ, Santolaria F, Martínez-Riera A, González-Reimers E, de la Vega Prieto M, et al. (2006) Clinical signifi- cance of homocysteine in elderly hospitalized patients. Metabolism Clinical and Experimental 55: 620-627.

28. Lee BJ, Huang MC, Chung LJ, Cheng $\mathrm{CH}$, Lin $\mathrm{KL}$, et al. (2004) Folic acid and vitamin $B_{12}$ are more effective than vitamin $\mathrm{B}_{6}$ in lowering fasting plasma homocysteine concentration in patients with coronary artery disease. European Journal of Clinical Nutrition 58: 481-487.

29. Andersson SE, Edvinsson M, Edvinsson L (2005) Reduction of homocysteine in elderly with heart failure improved vascular function and blood pressure control but did not affect inflammatory activity. Basic \& Clinical Pharmacology \& Toxicology 97: 306-301.

30. Schoenenberger AW, Schoenenberger-Berzins R, Auf der Maur C, Suter PM, Vergopoulos A, et al. (2012) Thiamine supplementation in symptomatic chronic heart failure: $A$ randomized, double-blind, placebo-controlled, cross-over pilot study. Clinical Research in Cardiology 101: 159-164.

31. Almoznino-Sarafian D, Sarafian G, Berman S, Shteinshnaider M, Tzue I, et al. (2009) Magnesium administration may improve heart rate variability in patients with heart failure. Nutrition, Metabolism \& Cardiovascular Diseases 19: 641-645.

32. Mantle D (2013) Pharmacology of coenzyme $Q_{10}$ : Relevance to cardiovascular and other disorders. Nurse Prescribing 11: 602-607.

33. Kumar A, Kuar H, Devi P, Mohan V (2009) Role of coenzyme $Q_{10}\left(\mathrm{CoQ}_{10}\right)$ in cardiac disease, hypertension and Meniere-like syndrome. Pharmacology \& Therapeutics 124: 259-268.

34. Herrmann M, Muller S, Kindermann I, Gunther L, Konig J, et al. (2007) Plasma B vitamins and their relation to the severity of chronic heart failure. The American Journal Clinical Nutrition 85: 117-123.

35. DiNicolantonio JJ, Niazi AK, Lavie CJ, O'Keefe JH, Ventura $\mathrm{HO}$ (2013) Thiamine supplementation for the treatment of heart failure: A review of the literature. Congestive Heart Failure 19: 214-222. 